\title{
Cultivos dendroenergéticos de híbridos de álamo para la obtención de biocombustibles en Chile: estado del arte
}

\author{
Short rotation woody crops of hybrid poplars for bioenergy in Chile: state of the art \\ Ricardo Baettig Pa*, Marco Yáñez A ${ }^{\mathbf{a}}$, Marta Albornoz A A $^{\mathbf{a}}$ \\ *Autor de correspondencia: aUniversidad de Talca, Centro Tecnológico del Álamo (CTA), \\ 2 Norte 685, casilla 747, Talca, Chile, rbaettig@utalca.cl
}

\begin{abstract}
SUMMARY
Because of the harmful effect on the environment and the uncertainty in the future offer of the conventional source of energy, the lignocellulosic biomass is being considered as the most promising alternatives of solution. The evidence shows that an important way to get biomass turned into biofuels will be through establishing plantations with high growth rates and short rotation periods; plantations specially conceived for that. This article describes characteristics and possibilities of using hybrid poplars on energetic plantation models, which has an enormous potential to be developed in Chile.
\end{abstract}

Key words: biomass, hybrid poplars, bioenergy.

\section{RESUMEN}

Frente a los efectos dañinos en el ambiente generados por el uso de combustibles fósiles, y debido a la incertidumbre en la oferta futura de las fuentes convencionales de energía, la biomasa lignocelulósica es considerada como una de las más importantes alternativas de solución. La evidencia señala que una importante vía para producir la biomasa utilizada en la generación de combustibles líquidos y sólidos será a través del desarrollo de plantaciones de rápido crecimiento y rotaciones cortas específicamente concebidas para la producción de biomasa. Por su parte, Chile no estará ajeno a la implementación de este tipo de tecnologías. En este artículo de revisión se describen las características y posibilidades del establecimiento de plantaciones energéticas utilizando híbridos del género Populus, género que presenta un gran potencial de desarrollo en el país.

Palabras clave: biomasa, híbridos de álamo, dendroenergía.

\section{INTRODUCCIÓN}

En la actualidad existe un amplio debate sobre la sustitución de energías derivadas de combustibles fósiles por el uso de energías renovables como la solar, eólica, geotérmica y la producida por la transformación de biomasa. Este debate nace en vista de los efectos dañinos generados por el uso de combustibles fósiles sobre el ambiente y por la incertidumbre en la oferta futura de las fuentes convencionales de energía. Anticipando una escasez de combustibles fósiles, tanto Europa como Estados Unidos se han fijado como objetivo obtener proporciones significativas de su energía a partir de fuentes biológicas. En la Unión Europea actualmente el $30 \%$ de la energía es utilizada en el transporte. De esta proporción, hoy un $98 \%$ proviene de combustibles fósiles, pero al año 2030 el objetivo es sustituir un $25 \%$ con biocombustibles (BIOFRAC 2006). Para ello, la Unión Europea ha establecido una estrategia que considera estimular la demanda de biocombustibles por medio del fomento a su producción y distribución. Dentro de esta estrategia se privilegiará la producción de biocombustibles de biomasa lignocelulósica o biocombustibles de segunda generación
(EU 2006). Por su parte, Estados Unidos ha establecido un objetivo al año 2030 de sustituir un $30 \%$ del petróleo por combustibles de origen biológico, para lo cual se requerirá un suministro anual de mil millones de toneladas anuales de biomasa (Perlack et al. 2005).

Desde el punto de vista de su apariencia física, los biocombustibles se clasifican en sólidos, líquidos y gaseosos (Camps y Marcos 2002). Los biocombustibles sólidos corresponden a los pellets, astillas y briquetas, así como la leña y el carbón vegetal. Los principales biocombustibles líquidos son el etanol o alcohol etílico, el metanol y el biodiesel. En cuanto a los biocombustibles gaseosos, éstos están formados por diferentes mezclas en que el monóxido de carbono $(\mathrm{CO})$, el metano $\left(\mathrm{CH}_{4}\right)$ y el hidrógeno $\left(\mathrm{H}_{2}\right)$ están presentes en forma preponderante. Los distintos biocombustibles son generados por la transformación de biomasa lignocelulósica, representada principalmente por los residuos de cultivos agrícolas como trigo (Triticum spp.) y maíz (Zea mays L.), entre otros, de la industria maderera (aserrín, despuntes, etc.), de la cosecha forestal (ramas) y la biomasa proveniente de cultivos intensivos dedicados a estos fines (plantaciones energéticas). 
En el caso de Chile, los combustibles usados por el transporte en la actualidad son exclusivamente de origen fósil y una proporción cercana al $60 \%$ de la electricidad es generada a partir de fuentes fósiles (CNE 2008). Chile alcanzó el cénit de producción de petróleo en la década de 1970 y, desde entonces, la producción nacional de petróleo ha ido decreciendo sostenidamente, hasta alcanzar hoy en día sólo un $2 \%$ del consumo. A su vez, la alta proporción de energía renovable que caracterizó la producción de electricidad hasta mediados de la década de 1990, sustentada en la operación de centrales hidroeléctricas, ha perdido relevancia frente a la puesta en marcha de centrales termoeléctricas usando combustibles fósiles. Esta producción termoeléctrica está seriamente amenazada en el futuro cercano por la imperiosa necesidad de importar combustibles que serán probablemente cada vez más escasos y seguramente más costosos.

Este artículo de revisión describe las características y posibilidades del establecimiento de plantaciones energéticas con el uso de híbridos del género Populus spp. (álamos), para la producción de biomasa y biocombustibles. Se pone énfasis en la aplicación de la tecnología en Chile, país donde el cultivo de híbridos de álamo podría tener un enorme potencial de desarrollo.

\section{BIOMASA LIGNOCELULÓSICA COMO BIOCOMBUSTIBLE}

Biomasa lignocelulósica como biocombustible sólido. La principal fuente de materia prima para obtener biocombustibles sólidos sigue siendo hasta hoy el acopio de los residuos de la industria de conversión de la madera, de cultivos agrícolas y de faenas de cosecha forestal. Considerando sólo este escenario, el país presenta un límite en la capacidad de producción de energía y sería equivalente a la capacidad de producción de residuos aprovechables energéticamente (RAE), ya sea de las cosechas de los bosques como de las industrias de conversión. De hecho, se estima que las 34.000 hectáreas de Eucalyptus spp. y las 70.000 hectáreas de Pinus radiata D. Don que se cosechan anualmente en Chile pueden proveer un total de 3,1 millones de toneladas de biomasa lignocelulósica, principalmente restos de fuste y ramas (Campino 2006).

El poder calorífico anhidro de la biomasa lignocelulósica es poco variable y se encuentra usualmente en la literatura un valor entre 4.400 y $4.600 \mathrm{kcal} \mathrm{kg}^{-1}\left(5,23 \mathrm{MWh} \mathrm{Mg}^{-1}\right)$ (Kauter et al. 2003). La biomasa presenta un contenido de humedad más alto mientras más fresca ésta sea. Con el aumento del contenido de humedad se produce una rápida pérdida de poder calorífico (Ince 1977). En el supuesto que los residuos de cosecha llegaran a una planta termoeléctrica con un contenido de humedad de $33 \%$ en base húmeda (50\% en base seca), ellos representarían un potencial energético de 10,4 millones de MWh al año. Considerando una eficiencia de conversión de $38 \%$ en la obtención de energía eléctrica, este potencial energético representa una planta termoeléctrica de $450 \mathrm{MW}$ funcionando los 365 días del año a plena capacidad. Estimaciones realizadas acerca del potencial energético del bosque nativo arrojan cifras más importantes aún (Campino 2006, Emanuelli 2006). Sin embargo, la dispersión geográfica de esta biomasa y la enorme variabilidad de sus cualidades hacen inciertos los costos de una industria basada en su acopio y posterior transformación.

En cuanto a los RAE de la industria de conversión mecánica, se estima un potencial de 3,2 millones de toneladas, conformadas principalmente de aserrín, corteza, despuntes y viruta (CNE/GTZ/INFOR 2007). Suponiendo que esta biomasa tiene un contenido de humedad promedio de $23 \%$ en base húmeda ( $30 \%$ en base seca) podría representar un potencial energético de 12,7 millones de MWh al año. Con una eficiencia de conversión de $38 \%$ en la obtención de energía eléctrica, este potencial energético representa una planta termoeléctrica de $550 \mathrm{MW}$ funcionando los 365 días del año a plena capacidad. En cifras redondas, el potencial de RAE provenientes de la industrialización de las plantaciones forestales chilenas representa una potencia eléctrica de $1.000 \mathrm{MW}$.

Biomasa lignocelulósica para la obtención de biocombustibles líquidos. Actualmente los biocombustibles líquidos son obtenidos de cultivos oleaginosos como raps (Brassica napus L.), maravilla (Helianthus annuus L.) y soya (Glycine $\max$ L. Merrill.), y de cultivo alcoholígenos como cereales (Triticum spp.), remolacha (Beta vulgaris L.) y caña de azúcar (Saccharum officinarum L.), biocombustibles denominados comúnmente como de primera generación. Por ejemplo, en Europa la mayor parte del bioetanol producido proviene del cultivo de cereales como trigo, cebada (Hordeum spp.) y el biodiesel del raps. Brasil, el segundo productor mundial de bioetanol, utiliza la caña de azúcar como fuente de azúcares simples. A su vez, Estados Unidos, actual primer productor mundial, utiliza el maíz como fuente de carbohidratos para abastecer la industria del bioetanol. Sin embargo, diversos estudios dan cuenta que los rendimientos energéticos de los biocombustibles de primera generación son pobres, obteniéndose un poco más de un litro de biocombustible por cada litro consumido en los procesos de establecimiento, labranza, cosecha y transformación (Farrell et al. 2006). Esto hace que para sustituir proporciones significativas del consumo de combustibles fósiles se requiera cultivar extensas regiones de territorio con altas demandas en cuanto a calidad de suelo, consumo de agua y de fertilizantes.

Por otra parte, existe consenso en que la obtención de biocombustibles de origen lignocelulósico (llamados también biocombustibles de segunda generación o BtL del inglés biomass to liquid) significará un salto extraordinario en los rendimientos energéticos. Se estima una relación de 1,8:1 hasta 36:1 en cuanto al rendimiento energético de biocombustible con respecto al consumo de combustibles 
fósiles en las etapas de cultivo, cosecha y transformación (Gnansounou y Dauriat 2005, Rutz y Janssen 2008). Sin embargo, hoy en día, la transformación química y bioquímica de la madera en bioetanol o en biodiesel no ha llegado a una fase de plena industrialización. El interés político de los principales países industrializados sobre el tema se ha materializado en importantes inversiones en investigación. Existen diversos procesos que están siendo estudiados y escalados a nivel prototipo. Según el reporte de BIOFRAC (2006), se estima que el desarrollo en gran escala de los BtL tendrá lugar en la década de 2020 a 2030 y este desarrollo consistirá de las siguientes fases:

- Fase inicial (hasta 2010): mejora de tecnologías en biocombustibles de primera generación, construcción de plantas demostrativas de BtL.

- Segunda fase (2010 a 2020): desarrollo de plantas comerciales de BtL y de cultivos energéticos específicos para esta finalidad en el marco de una agricultura sostenible.

- Fase de consolidación (desde 2020): producción de BtL a gran escala, creación de complejos agroindustriales con integración del componente transformador y de la producción de biomasa.

\section{PLANTACIONES FORESTALES PARA ENERGÍA}

Marco conceptual de las plantaciones para energía. Como una manera de contribuir a la reducción del aumento del efecto invernadero y de reducir la dependencia energética de combustibles importados, países desarrollados como Estados Unidos, Suecia, Italia, Holanda y España están desarrollando plantaciones específicamente concebidas para la producción de biomasa con altas tasas de acumulación anual y cortos períodos de rotación (Volk et al. 2004, Dimitriou y Aronsson 2005, Marcos 2006). Esto se logra en plantaciones de alta densidad entre individuos, usando genotipos mejorados y una alta intensidad en actividades silviculturales como la fertilización, el riego y control de la competencia (Johnson et al. 2007). Este tipo de cultivos forestales se conoce usualmente como SRWC, por su denominación en inglés de short rotation woody crops o como WPEC de woody perennial energy crops. Los géneros de especies forestales más frecuentemente utilizados son el Populus, al cual pertenecen los álamos (Riemenschneider et al. 2001, Coleman et al. 2004b, Ragauskas et al. 2006) y el Salix, constituido por los sauces (Smart et al. 2005). Estos géneros no sólo poseen un enorme potencial de generación de biomasa, sino que se adaptan a una amplia variedad de condiciones edafoclimáticas, presentan facilidad de propagación y por ende de escalamiento de plantaciones, alta capacidad de rebrote, son objeto de silvicultura clonal intensiva, de programas de mejoramiento genético y de estudios biotecnológicos avanzados. Prueba de ello es que Populus trichocarpa (Torr. et Gray) ha sido la primera especie forestal y la tercera del reino vegetal después del arroz (Oryza sativa L.) y el Arabidopsis thaliana (L.), Heynh., cuyo genoma haya sido secuenciado (Tuskan et al. 2006).

Modelos de plantación y rendimientos. La densidad de plantación tiene una enorme influencia en la rapidez de acumulación de biomasa por hectárea. Una mayor densidad de plantación se traduce en una mayor cantidad de individuos formando biomasa, sin embargo, una cantidad excesiva de individuos frenará inevitablemente el crecimiento de los árboles por competencia por luz a nivel de las copas, como por la captura de agua y de nutrientes en las raíces. Indudablemente, la productividad de la plantación a altas densidades está relacionada con la tolerancia que puedan presentar las especies a una alta competencia por recursos, la fertilidad del suelo y las características climáticas del sitio. La bibliografía describe rangos extraordinariamente amplios que van desde 1.500 a más de 100.000 individuos por hectárea (DeBell et al. 1997, Stanton et al. 2002, Marcos 2006). A principios de la década de 1980 fue propuesto en Estados Unidos un modelo de espaciamiento entre plantas de 0,18 a $0,30 \mathrm{~m}$, obteniéndose una densidad de plantación entre 100 mil y 600 mil individuos por hectárea (híbrido D-01 de Populus $s p$. de un taxón desconocido). Este modelo fue llamado "woodgrass" y con base en cosechas anuales se pretendía que era posible obtener rendimientos superiores a $100 \mathrm{Mg}$ ha $^{-1}$ año $^{-1}$ (DeBell et al. 1997). No obstante, ensayos de rendimiento arrojaron tasas de incremento en biomasa de sólo $7 \mathrm{Mg} \mathrm{ha}^{-1}$ año $^{-1}$ con máximos de hasta 9,6 Mg $\mathrm{ha}^{-1}$ año $^{-1}$ alcanzados durante el segundo año. En estos ensayos se obtuvieron altas tasas de mortalidad, de hecho, al cabo de cinco años la proporción de plantas sobrevivientes alcanzó un $30 \%$ para el espaciamiento de $0,18 \mathrm{~m}$ y de $50 \%$ para el espaciamiento de $0,30 \mathrm{~m}$ (ensayo del híbrido H-11 de taxón P. trichocarpa $x$ P. deltoides Bartr.) (DeBell et al. 1997).

En la década de 1990, diferentes países comienzan a desarrollar masivamente ensayos SRWC para estimar rendimientos de biomasa. Las densidades de plantación siguen cubriendo un amplio rango que va de 1.500 (Stanton 2001) hasta 36.000 individuos ha ${ }^{-1}$ (Marcos 2006). En el oeste norteamericano se reconoce un modelo de espaciamientos medios, con densidades de 1.500 a 2.200 individuos ha- ${ }^{-1}$, en rotaciones de cinco a siete años. Este modelo ha sido llamado "modelo norteamericano" o MRF (medium rotation forestry); se estudia en Estados Unidos con híbridos del género Populus spp. y también en Europa, aunque en menor medida. El llamado "modelo europeo" o SRF (short rotation forestry) consiste en densidades de plantación desde unos 5.000 individuos ha ${ }^{-1}$ hacia arriba, en períodos de rotación usuales de dos años. Este modelo se estudia en la mayor parte de los países de Europa y en el noreste de Estados Unidos usando principalmente híbridos de Populus spp. e híbridos de Salix spp. Las principales ventajas y desventajas de ambos modelos se resumen en el cuadro 1. Sin 
embargo, la aplicación y conveniencia de un modelo u otro dependerá de los rendimientos de determinados híbridos en sitios específicos, los costos de cada una de las faenas y la calidad del producto requerida.

Con el modelo MRF se cosechan árboles de un mayor tamaño que en SRF, lo cual presenta ventajas en la flexibilidad de la plantación así como en la calidad de la composición química de la biomasa. En árboles más pequeños la proporción que representa la corteza del árbol en el total de biomasa es mayor, lo que afecta las transformaciones posteriores. Por ejemplo, la madera de Populus spp. presenta alrededor de 0,2\% de su peso en nitrógeno, mientras que la corteza alrededor de un $1 \%$, lo cual se traduce en una mayor emisión de óxidos de nitrógeno $\left(\mathrm{NO}_{\mathrm{x}}\right)$ en la combustión de biomasa incluyendo corteza (Kauter et al. 2003).

En el primer año de establecimiento de una plantación a alta densidad, la planta experimenta un crecimiento limitado debido a la poca extensión de su sistema radicular y de su superficie fotosintética, situación que mejora considerablemente en el segundo año hasta que se completa el ciclo definido para la corta. Por otra parte, entre ciclos sucesivos de corta se produce una mayor tasa de generación de biomasa debido al número de rebrotes desde la cepa. El rebrote presenta un crecimiento aún más alto que la plantación inicial, debido a que las cepas ya tienen un sistema radicular desarrollado y se consigue un control más eficaz de la competencia herbácea (Sixto et al. 2007). La mantención del vigor de las cepas, a través de sucesivos rebrotes, depende de factores como el genotipo, su interacción con el sitio y con las prácticas silviculturales aplicadas (Sixto et al. 2007). En general, en SRF la mayor tasa de incremento en biomasa se alcanza entre el tercer y el quinto año (DeBell et al. 1997), sin embargo, el punto óptimo en que resulta conveniente la cosecha dependerá de una interrelación compleja de factores fisiológicos, técnicos y económicos. Por ejemplo, la cosecha de árboles muy grandes es más costosa y el manejo de rebrotes es más ineficiente. Se supone que se podrían alcanzar entre cuatro y siete rotaciones bienales sin renovación de plantas, es decir, las cepas llegarían a una edad de ocho a catorce años cuando deban ser retiradas y replantado el terreno (Marcos 2006, Sperandio 2007). De hecho, Pontailler et al. (1999) estudiaron el rendimiento en biomasa en cuatro híbridos de álamo durante cinco ciclos de dos años de crecimiento, encontrando que la capacidad de generación de brotes de las cepas no se vio afectada después de 10 años de rotaciones de intervalos cortos.

Selección de híbridos de álamo para plantaciones dendroenergéticas. El género Populus presenta alrededor de una treintena de especies adaptadas a una gran diversidad de condiciones edafoclimáticas, lo que revela una amplia plasticidad genética (Zamudio et al. 2008). La habilidad del género Populus para producir hídridos intra e interespecies, tanto en forma espontánea como controlada, posibilita la creación de un gran número de subespecies e híbridos simples y complejos (Orlovic et al. 1998).

La mayor parte de las plantaciones comerciales ha sido realizada con híbridos provenientes de cruzamientos interespecíficos. Los híbridos mejor estudiados corresponden a cruzamientos $P$. trichocarpa $x P$. deltoides (por ejemplo: Hazendans, Hoogvorst, Beaupré, Raspalje, Boelare) y P. deltoides $x$ P. nigra L. (por ejemplo: I-214, Campeador, Dorskamp). Con respecto a los rendimientos en biomasa, Sixto et al. (2007) presentan una detallada revisión de investigaciones realizadas en diversos países, con diferentes híbridos y densidades de plantación. La tasa promedio anual de incremento en biomasa muestra un amplio rango, variando desde sólo $3 \mathrm{Mg} \mathrm{ha}^{-1}$ año $^{-1}$ (con un híbrido TxD ensayado a 10.000 cepas ha $^{-1}$ en Bélgica) hasta $35 \mathrm{Mg} \mathrm{ha}^{-1}$ año $^{-1}$ (con otro híbrido TxD a la misma densidad de plantación, pero en Estados Unidos). Debido a la diversidad de condiciones de estos estudios se hace muy difícil realizar comparaciones, sin embargo, varias de las referencias señalan rendimientos superiores a 20 $\mathrm{Mg} \mathrm{ha}^{-1} \mathrm{año}^{-1}$. En Italia, recientemente se han inscrito una serie de nuevos híbridos orientados a la producción de biomasa, tales como: Monviso, Pegaso, Saturno, Marte y Sirio (Facciotto et al. 2006). La figura 1 presenta los

Cuadro 1. Ventajas y desventajas de los principales modelos de plantación dendroenergética.

Advantages and disadvantages of the main models of SRWC.

\begin{tabular}{|c|c|c|}
\hline Modelo & Ventajas & Desventajas \\
\hline $\begin{array}{l}\text { SRF o "modelo europeo", } 5.000 \\
\text { a } 36.000 \text { plantas } \text { ha }^{-1} \text {. } \\
\text { Cortas cada uno a tres años con } \\
\text { rebrote. }\end{array}$ & $\begin{array}{l}\text { - Alto rendimiento en producción de } \\
\text { biomasa. } \\
\text { - Costos de cosecha más bajos. }\end{array}$ & $\begin{array}{l}\text { - Elevados costos de plantación. } \\
\text { - Poca flexibilidad de productos. } \\
\text { - Menor calidad de biomasa, mayor proporción de } \\
\text { corteza, mayor contenido de humedad. } \\
\text { - Mayor tendencia a la formación de patologías. }\end{array}$ \\
\hline $\begin{array}{l}\text { MRF o "modelo americano", } \\
1.000 \text { a } 2.000 \text { plantas } \text { ha }^{-1} \text {. }\end{array}$ & $\begin{array}{l}\text { - } \text { Menores costos de plantación. } \\
\text { - } \text { Mayor flexibilidad de productos } \\
\text { (pulpa, pallets, energía). } \\
\text { - } \text { Mejor calidad de la biomasa, menos } \\
\text { corteza, menos humedad. } \\
\text { - Control más fácil de patologías. }\end{array}$ & $\begin{array}{l}\text { - Menor rendimiento en producción de biomasa. } \\
\text { - Rotaciones más largas. } \\
\text { - Costos de cosecha más altos. }\end{array}$ \\
\hline
\end{tabular}


rendimientos en biomasa anhidra obtenidos en ensayos de SRF en densidades de plantación de alrededor de 7.500 individuos ha $^{-1}$ para nuevos y antiguos híbridos italianos: Monviso (DTxN), AF2 (DxN), AF6 (DTxN), AF8 (DTxT), I-214 (DxN) y Lux (D). Estos ensayos fueron realizados en la región de Venecia a una latitud de $45^{\circ}$ Norte. Se pueden apreciar diferencias en el rendimiento de biomasa seca por hectárea entre los diferentes híbridos, especialmente para la segunda rotación; híbridos como el Monviso y el AF8 pueden alcanzar un $70 \%$ más de rendimiento respecto de híbridos como Lux. La variabilidad en la producción de biomasa de diferentes tipos de híbridos de álamos muestra las posibilidades de selección genética como medio para aumentar la productividad.

En cualquiera de los modelos silviculturales mencionados anteriormente, la rentabilidad de la plantación estará condicionada principalmente por el rendimiento en biomasa con características deseables. La producción de biomasa es un carácter complejo en el que intervienen componentes morfológicos, fisiológicos y bioquímicos que son regulados por genes (Yu 2001). Por lo tanto, la selección genética es uno de los métodos por los cuales se puede aumentar el rendimiento de un cultivo (Riemenschneider et al. 2001).

Una lista de características fisiológicas y de crecimiento importantes para ser incluidas en un programa de selección son documentadas por Corcuera et al. (2005). En SRWC casi siempre se busca incrementar la tasa de

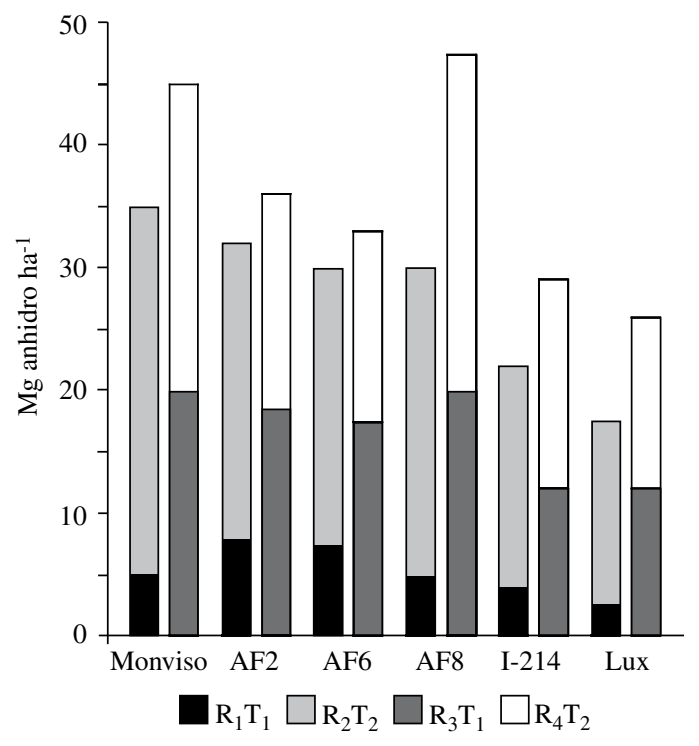

Figura 1. Rendimiento en biomasa de seis híbridos de Populus $s p$. en rotaciones bienales. Ri indica la edad del sistema radicular y $\mathrm{Tj}$ la edad del tallo. Primera rotación $\left(\mathrm{R}_{1} \mathrm{~T}_{1}\right.$ y $\left.\mathrm{R}_{2} \mathrm{~T}_{2}\right)$ y segunda rotación $\left(R_{3} T_{1}\right.$ y $\left.R_{4} T_{2}\right)$. Fuente: Nardin (2007).

Annual increment of biomass of six hybrid poplars by biannual rotations. $R_{i}$ indicates the age of the root system and $T_{j}$ the age of the stem. First rotation: $\left(\mathrm{R}_{1} \mathrm{~T}_{1}\right.$ and $\left.\mathrm{R}_{2} \mathrm{~T}_{2}\right)$ and second rotation: $\left(\mathrm{R}_{3} \mathrm{~T}_{1}\right.$ and $\mathrm{R}_{4} \mathrm{~T}_{2}$ ). Source: Nardin (2007). generación de biomasa por medio de una mejora en la tasa de fotosíntesis, en la respuesta al fotoperíodo, una optimización de la arquitectura de hojas y ramas, una mayor resistencia a agentes bióticos y tolerancia a estrés abióticos, una esterilidad floral, una caída de hojas tardía, un crecimiento más bien en grosor que en altura, una absorción mejorada de nitrógeno y una mayor eficiencia en el uso de los nutrientes (Johnson et al. 2007). Para el género Populus se encuentra ampliamente documentada una alta variabilidad en características de crecimiento y de fenotipo que puede ser aprovechada con fines de mejoramiento genético (Ceulemans et al. 1990, Deraedt y Ceulemans 1998, Rae et al. 2004). Estos autores señalan que las características que más influyen sobre el rendimiento en biomasa son el crecimiento en altura, el diámetro basal, el número de brotes y número de ramas silépticas. No obstante, aparte de las correlaciones entre diferentes características fisiológicas y morfológicas con la producción de biomasa, la ganancia genética final de un proceso de mejoramiento genético dependerá del grado de control genético de estas características. También, ciertos híbridos pueden cambiar drásticamente su comportamiento fenotípico dependiendo de las condiciones de sitio y manejo, por lo cual es indispensable tener en cuenta el efecto de la interacción genotipo-ambiente en los programas de mejoramiento orientados a producción de biomasa. En el caso de Chile, debido a la diversidad de condiciones fisiográficas y climáticas, el efecto de la interacción genotipo-ambiente determina un componente particularmente delicado en la selección de genotipos para fines energéticos.

En Chile la variedad genética de los álamos era extremadamente reducida, de hecho, según la Comisión Nacional del Álamo, se acepta que la gran mayoría de las plantaciones de álamos actuales en Chile, establecidas con fines industriales, ha sido generada mediante propagación vegetativa a partir de no más de seis híbridos (Zamudio et al. 2008). Considerando la poca diversidad genética y, por otro lado, el enorme potencial que podría tener el género Populus en Chile, tanto para la industria de la madera como con fines energéticos, en el año 1999 el Centro Tecnológico el Álamo (CTA - Universidad de Talca) comenzó la importación y ensayo de alrededor de 2.500 híbridos experimentales generados por la Poplar Molecular Genetics Cooperative (PMGC - Universidad del Estado de Washington), a partir de cinco especies de Populus. Mediciones realizadas en diferentes niveles de ensayo clonal establecidos por el CTA, entre las regiones de O'Higgins y de Los Ríos, indican que varios de estos híbridos tienen potencial para generar incrementos en biomasa anhidra que superarían $30 \mathrm{Mg} \mathrm{ha}^{-1} \mathrm{año}^{-1}$. Paralelamente, el año 2001, el CTA realizó la introducción al país de 20 híbridos pertenecientes al catálogo europeo e importados desde Francia. Estos híbridos fueron generados a partir de dos especies europeas ( $P$. alba L. y $P$. nigra) y dos norteamericanas $(P$. deltoides y $P$. trichocarpa), los cuales 
son utilizados comercialmente desde hace varios años. En el año 2004, otra importación fue realizada con híbridos de la empresa Greenwood Resources Inc., originados de padres de $P$. trichocarpa y $P$. deltoides.

Resultados preliminares obtenidos por el CTA, que no han sido aún publicados, muestran la presencia de interacción genotipo-ambiente, es decir, muchos híbridos cambian su comportamiento y potencial según las condiciones específicas de algunos sitios. Sin duda, el éxito de un programa de plantaciones dendroenergéticas con híbridos de álamos para Chile dependerá de la implementación de una amplia red de ensayos clonales, con diseños experimentales específicos para la selección de genotipos superiores.

Dinámica del carbono en los suelos de cultivo. El carbono orgánico del suelo afecta la mayoría de las propiedades químicas, físicas y biológicas del suelo vinculadas con su calidad, sustentabilidad y capacidad productiva (Martínez et al. 2008). Dependiendo de su uso, el suelo actúa ya sea como fuente o como fijador de carbono. Globalmente, los suelos contienen más carbono que la suma de carbono existente en la vegetación y en la atmósfera (Swift 2001). El carbono orgánico del suelo ocupa el 69,8\% del carbono orgánico en la biosfera (Robert 2002). Los ecosistemas forestales contienen más carbono por unidad de superficie que cualquier otro tipo de uso de la tierra. Estos suelos, que cubren el $29 \%$ de las tierras, contienen el $60 \%$ del carbono de la vegetación terrestre y el $40 \%$ del total de carbono (Robert 2002). La captura de carbono en los suelos es una promesa para la mitigación del cambio climático. El potencial de la agrosilvicultura, del manejo de bosques y el mejoramiento de las prácticas agrícolas y de pastoreo podría permitir la captura de $0,53 \mathrm{Pg}$ de carbono al año, equivalentes a un $10 \%$ de las emisiones de combustibles fósiles (Robert 2002).

Para los cultivos dendroenergéticos el carbono acumulado en las temporadas de crecimiento (biomasa aérea formada por fotosíntesis) es reemitido como $\mathrm{CO}_{2}$ atmosférico en tan sólo unas semanas o meses luego de la cosecha, mediante los procesos de combustión directa o de conversión y combustión de biocombustibles líquidos. Descartando otras emisiones propias a la silvicultura, existe un balance neutro. En tanto esta dendroenergía sirve para desplazar consumo de combustibles fósiles, se produce una contribución positiva a la reducción de emisiones de carbono (Tuskan y Walsh 2001). Hoy en día existe controversia acerca de la capacidad de almacenamiento de carbono en los suelos dedicados a los cultivos dendroenergéticos. Los estudios de este tipo todavía entregan valores disímiles y dependen fuertemente de la condición inicial a la cual estaba sometido el suelo antes del establecimiento del cultivo. Por ejemplo, Dowell et al. (2008) realizaron un perfil de variación de carbono en la profundidad del suelo en el transcurso de cinco años y señalan una pérdida de carbono orgánico de $22 \mathrm{Mg} \mathrm{ha}^{-1}$ en el período estudiado. El empobrecimiento de carbono estaría sobre todo ocurriendo en los 12,5 primeros centímetros de suelo. Asimismo, Hansen (1993) da cuenta de una pérdida de carbono orgánico en los primeros años postestablecimiento de una plantación dendroenergética con híbridos de álamo. Sin embargo, Coleman et al. (2004a) encuentran un leve incremento transcurridos los primeros 40 meses de establecimiento de la plantación dendroenergética de álamos. Se señala que los mayores aumentos ocurren en los suelos más pobres, marginales para la agricultura (Coleman et al. 2004a), lo cual abre expectativas para que Chile pueda usar parte de la amplitud de suelos que han sido sobreexigidos por la agricultura durante décadas. Por otra parte, Schlatter y Gerding (2001) señalan que en Chile la mayoría de las plantaciones forestales actuales disminuyen la cantidad de materia orgánica del suelo, debido a las prácticas de preparación del suelo y a la quema de desechos realizada después de la cosecha a tala rasa de las plantaciones. Cabe destacar que en los cultivos dendroenergéticos estas prácticas no son utilizadas debido a que produce un aprovechamiento completo de la biomasa aérea y al aprovechamiento de la capacidad de rebrote. Sin embargo, en un contexto más amplio, habría que considerar otras emisiones ligadas al consumo energético realizados durante la fabricación de pesticidas, herbicidas, fertilizantes, lubricantes, insumos variados, emisión directa por quema de combustibles durante el laboreo, consumo energético del bombeo de agua de riego, entre muchas otras.

Mecanismos de masificación y establecimiento. La masificación mediante reproducción vegetativa para lograr un escalamiento de estas plantaciones de alta densidad podría llegar a configurar una dificultad. Por ejemplo, el sistema de masificación de álamos tradicionalmente usado en Chile mediante varas da muestras de clara obsolescencia si se le compara en productividad con los sistemas de reproducción por estacas de un largo entre 20 y $45 \mathrm{~cm}$ que se usan en países que están avanzados en SRWC. Estas estacas son cortadas desde de tallos de un año de edad y son obtenidas y plantadas durante el estado de dormancia, es decir, enraízan y brotan en el sitio de plantación (Stanturf et al. 2001).

Uno de los pasos importantes en el éxito del establecimiento de plantaciones de álamos es la preparación de los sitios (Hansen et al. 1983), la cual incrementa la productividad principalmente por la reducción de malezas en la etapa de plantación (Thomas et al. 2000), mejora la capacidad para la exploración de las raíces y facilita en definitiva el establecimiento de la planta. La preparación de suelos es específica para cada sitio y dependerá del relieve, textura, historial de manejo, tipo de malezas presentes, etc. y debe ser acorde al tipo de material vegetativo que se utilizará en la plantación, el cual varía desde estacas no enraizadas de 20 a $45 \mathrm{~cm}$ de longitud hasta varas de 2 a $4 \mathrm{~m}$. En general, los sitios para un óptimo desarrollo serán aquellos que presenten buena aireación, suficiente contenido de humedad y nutrientes, que sean profundos, 
de textura media (arena y limo) y con un rango de $\mathrm{pH}$ entre 5,0 y 7,5 (Baker y Broadfoot 1979). Sin embargo, la presencia de interacción genotipo-ambiente permite ampliar el rango de adaptación y productividad a través de la selección genética y la tecnología silvicultural, posibilitando la incorporación de suelos y condiciones de menor calidad.

En los sistemas tradicionales de plantación de álamos, las varas son insertadas y fijadas manualmente con la ayuda de palas. El agujero de plantación con una profundidad cercana a un metro es normalmente realizado por una ahoyadora mecanizada. Dada la alta densidad de las plantaciones dendroenergéticas, resulta prácticamente obligatoria la mecanización de las faenas de plantación. En el caso del modelo norteamericano se usan varas que son insertadas en una zanja realizada por una herramienta de arado tirada por un tractor agrícola de por lo menos $90 \mathrm{~kW}$. En el caso del modelo europeo, se utilizan distintos tipos de máquinas plantadoras de estacas movidas por un tractor agrícola con potencia de entre 40 y $60 \mathrm{~kW}$. Estas máquinas hacen penetrar las estacas por medio de un dispositivo hidráulico en un terreno previamente preparado para tales efectos. Usualmente la preparación del suelo consiste en un arado hasta por lo menos $40 \mathrm{~cm}$ de profundidad, seguido de una homogeneización. El rendimiento de las máquinas plantadoras oscila entre 0,3 y 0,7 ha $\mathrm{h}^{-1}$, dependiendo principalmente de la capacidad para plantar una hilera o dos hileras simultáneamente (Balsari et al. 2007).

Manejo silvicultural. El manejo silvicultural de plantaciones energéticas incluye control de malezas, riego y ocasionalmente fertilización. En la actualidad, todas estas actividades cuentan con un alto grado de desarrollo tecnológico y resulta posible la aplicación de una silvicultura de precisión. Las malezas compiten por agua y luz, disminuyendo el crecimiento de las plantas y provocando mortalidad (Stanturf et al. 2001). En la mayoría de los sitios, entre el 75 y $95 \%$ de las raíces de álamos y sauces se encuentra dentro del horizonte A (Crow y Houston 2004), donde las malezas obtienen la totalidad de los recursos que necesitan del suelo. Existen varios antecedentes de estrategias de control de malezas con herbicidas (Hansen y Netzer 1985, Netzer y Hansen 1992, Miller y Bloese 2002). En SRWC el control de malezas se lleva a cabo mediante el uso de herbicidas, en forma mecánica o en una combinación de ambos. Se ha encontrado que el crecimiento de híbridos de álamo fue un $70 \%$ mayor en parcelas tratadas con glifosato versus las parcelas testigo (Netzer y Hansen 1992). Sin embargo, los álamos son sensibles a muchos de los herbicidas de mayor uso, por lo que en algunos casos se prefiere el control mecánico a través de rastras y cultivadores. Por otro lado, el uso de herbicidas preemergentes reduce el potencial de mortalidad asociado con el uso de herbicidas postplantación, pero estos tienen un mayor costo (Welham et al. 2007). En general, la definición de una estrategia de control de malezas debe ser acorde con el tipo de malezas presente y la normativa vigente respecto al uso de agroquímicos, asegurando la sustentabilidad de estas actividades.

Por otro lado, existe controversia de la respuesta de los álamos a la fertilización de plantaciones energéticas. Por ejemplo, Welham et al. (2007) señalan que la fertilización no se justifica económicamente en suelos fértiles y que las mejores respuestas a la fertilización se encuentran en sitios pobres. Deficiencias de nitrógeno, fósforo y azufre pueden limitar considerablemente el crecimiento de los álamos (Brown y Driessche 2002). Aunque la fertilización puede aumentar la concentración de nutrientes en los diferentes tejidos, debe existir un cierto balance entre los diferentes nutrientes, si otro nutriente es deficiente o algún otro factor es limitante, puede no haber respuesta a la fertilización nitrogenada (Kauter et al. 2003, Coleman et al. 2006). Características del suelo como el historial de manejo, textura, materia orgánica, $\mathrm{pH}$ y humedad interactúan afectando la disponibilidad de nutrientes para las plantas. Cualesquiera sean las características del sitio, bajo un sistema de plantación energética, la remoción continua de biomasa en ciclos cortos hace necesario reponer el suministro de nutrientes vía fertilización para poder mantener la productividad del suelo y las tasas de producción. Hansen et al. (1988) señalan que para obtener un buen crecimiento en plantaciones de álamo debe mantenerse como mínimo un $3 \%$ de concentración de nitrógeno en hojas recientemente maduras. Como la respuesta y eficiencia en la fertilización depende de las características del sitio, del tiempo y método de aplicación y del tipo de fertilizante, la dosificación debe ser prescrita con la ayuda de análisis de suelo y foliar.

Con respecto al riego, la mayor parte de los álamos se caracterizan por su marcada hidrofilia, aun cuando se han encontrado especies de Populus desarrollándose favorablemente en ambientes con muy escasa pluviometría (Carle y Ma 2005). Esto es prueba que existen diferencias genéticas en el uso eficiente de agua y en la capacidad de tolerar estrés de sequía. Monclus et al. (2006), estudiando 29 híbridos de P. deltoides x P. nigra, encontraron que los genotipos más productivos tuvieron una más baja tolerancia a la sequía, mientras que los genotipos menos productivos presentaron un amplio rango de tolerancia a la sequía. Esto principalmente es debido a las diferencias morfológicas que existen entre diferentes genotipos, como son la densidad y tamaño de los estomas, y el tamaño y distribución de las hojas.

Respecto a la demanda de agua en plantaciones de álamo existen varios antecedentes (Madison y Brubaker 1996, Montoya 1988, Sixto et al. 2007), los cuales señalan que los suministros de riego van entre 2.000 y $8.000 \mathrm{~m}^{3}$ $\mathrm{ha}^{-1} \mathrm{año}^{-1}$. No obstante, como se mencionó anteriormente, existen variables que deben ser consideradas para determinar los niveles óptimos de riego. Las necesidades de riego para un crecimiento óptimo de plantaciones de álamo varía con 
la textura del suelo, drenaje del suelo, determinación de la "lámina neta" y con factores climáticos locales (Shock et al. 2002). Además, los efectos de variar la frecuencia de riegos o la cantidad de agua aplicada por riego sobre el requerimiento de agua total dependerán del tipo de suelo y del sistema empleado (Shock et al. 2002). Al igual que en cultivos agrícolas y frutales, en especies forestales los requerimientos de agua dependerán del estado fenológico y de la edad del cultivo. Gochis y Cuenca (2000) encontraron que el coeficiente de cultivo de los álamos es bajo al comienzo del período de crecimiento, incrementándose con el desarrollo y luego declinando hacia el término de la estación de crecimiento. Sus resultados encontraron un máximo coeficiente de cultivo que bordeó $0,44,0,66$ y 0,82 para plantaciones de uno, dos y tres años, respectivamente. Por otro lado, un estudio de la relación entre la producción de biomasa y el uso de agua en tres híbridos de Populus $s p$. mostró para los híbridos Beaupré y Trichobel una tasa de $4,4 \mathrm{~g}$ de biomasa por litro de agua evapotranspirada, mientras que el híbrido Ghoy mostró una tasa $3,5 \mathrm{~g} \mathrm{dm}^{-3}$, y estas relaciones fueron constantes durante los dos años de ensayo (Souch y Stephens 1998).

En el caso del control fitosanitario, es preferible abordarlo desde el punto de la selección genética de híbridos resistentes a los distintos agentes patógenos. Los álamos son susceptibles al ataque de diversos insectos, hongos, bacterias, virus e incluso vegetales superiores. Sin embargo, actualmente en Chile sólo dos enfermedades pueden llegar a ser causa de pérdidas económicas importantes, sobre todo en plantaciones de bajo espaciamiento, en donde se crean microclimas propicios para su desarrollo. Una de ellas es provocada por Melampsora larici-populina Kleb, cuyos principales huéspedes son los álamos de las secciones Aigeiros y Tacamahaca. Este hongo provoca la pérdida prematura del follaje, disminuyendo la capacidad fotosintética de la planta, provocando una reducción en crecimiento de entre 30 y $60 \%$ (Widin y Schipper 1981, May de Mio et al. 2006, Pinon et al. 2006). El desarrollo de esta enfermedad se hace más intenso al tratarse de cultivos monoclonales, además, depende del tiempo inicial de infestación, susceptibilidad clonal, localidad y condiciones climáticas en donde se establecen los cultivos (Spiers y Hopcroft 1988).

Por otro lado, Chaitophorus leucomelas Koch o pulgón del álamo es una plaga que está ampliamente distribuida en muchos países. En Chile ha dañado diversas especies de álamos (Ramírez et al. 2004), afectando la capacidad fotosintética del árbol, debido a la caída de hojas, causando una disminución considerable del crecimiento. Sin embargo, no se han realizado estudios que cuantifiquen dichas pérdidas. En general, las pestes más graves que afectan a los álamos a nivel mundial no han sido introducidas en Chile y se consideran plagas cuarentenarias. Por ejemplo, Venturia populina Vuill., Melampsora medusae Thiim, Mycosphaerella populorum G. E. Thompson son de gran importancia económica al ser agentes nocivos para la productividad del género, por lo que se deben tomar las precauciones necesarias para que no sean introducidas al país.

Cosecha y logística. La cosecha para bioenergía, tanto para sauces como para álamos, se basa en cortas al segundo o tercer año, durante el período de receso vegetativo, luego del cual el rebrote de tocón o cepa forma un nuevo individuo y el proceso es repetido por varias rotaciones (Abrahamson et al. 2002, Marcos 2006) procurando que la cepa que origina los rebrotes se mantenga sana y vigorosa. La maquinaria es adaptada y no se parece a los sistemas de cosecha forestal tradicional, puesto que los árboles poseen un tamaño reducido, tanto en diámetro como en peso. Los sistemas de cosecha más productivos están adaptados para extraer en forma rápida y eficiente la biomasa mediante un volteo, astillado y carguío prácticamente simultáneo, realizado por un cabezal de cosecha y astillado que va montado en un tractor agrícola (Abrahamson et al. 2002). El consorcio italiano CNER (Consorzio Nazionale Energie Rinnovabili Agricole) ha desarrollado un cabezal de cosecha especialmente adaptado para álamos de 8 a $10 \mathrm{~cm}$ de diámetro. Este dispositivo se comercializa bajo la denominación de GBE1 (Biomasse Europe 1) y se adapta a un tractor Claas-Jaguar que tiene una potencia de alrededor de $340 \mathrm{~kW}$ (Dal Re et al. 2007). Otra alternativa es el cabezal marca Spapperi, el cual puede cortar fustes de hasta $180 \mathrm{~mm}$ de diámetro y se adapta a la toma de fuerza de un tractor cosechando una hilera a la vez y avanzando en marcha reversa (Pari 2007). Otros sistemas están basados en un volteo de las hileras realizado por una sierra circular adosada a un tractor agrícola, seguido de un apilamiento provisorio y un posterior astillado en el bosque (Facciotto y Mughini 2007). Por otra parte, en plantaciones dendroenergéticas de rotaciones medias o modelo norteamericano se realiza la cosecha mediante sistemas tradicionales usando "feller-buncher".

Por otro lado, la cosecha estacional genera la necesidad de almacenamiento para asegurar un abastecimiento continuo a la industria de transformación. En el caso de biocombustibles para la generación de electricidad, esta dificultad logística constituye una oportunidad para reducir el contenido de humedad de la biomasa, mejorando sustancialmente sus propiedades energéticas. En cuanto a los factores limitantes de la eficacia de secado de los residuos destacan: la humedad relativa ambiental, las temperaturas medias y extremas, el régimen de precipitaciones, la intensidad de los vientos dominantes, el grado de insolación y la exposición. En el interior de las pilas se producen fermentaciones parcialmente anaerobias y se desarrollan bacterias y esporulan hongos, lo cual produce un importante incremento de temperatura que, por encima del límite de $60^{\circ} \mathrm{C}$, ocasiona degradación química de los compuestos lignocelulósicos. En casos extremos se puede llegar a producir incluso la autoignición, riesgo que aumenta cuando se hacen montones de astillas demasiado 
grandes. Se han estudiado mecanismos para acelerar el proceso natural de secado, como por ejemplo la inducción de un efecto chimenea, en que el calor y el aire húmedo son evacuados hacia la cima de las pilas por medio de la introducción de tubos. Otra posibilidad es el uso de materiales textiles semiimpermeables que permiten la evaporación de la humedad protegiendo al mismo tiempo de la lluvia (Nardin 2007).

\section{CONCLUSIONES}

En países desarrollados la biomasa lignocelulósica proveniente de cultivos energéticos está empezando a visualizarse como una de las mejores opciones para mitigar la emisión de gases que provocan el cambio climático y la escasez futura de fuentes de energía. Para el caso de la producción de biomasa con especies forestales, la tendencia avalada por distintos estudios apunta a la aplicación de cultivos con altas densidades de plantación con rotaciones cortas bajo sistemas intensivos de cultivo.

Todavía existen muchas interrogantes acerca de las especies y genotipos adecuados, espaciamiento, edad de cosecha y tratamientos silviculturales óptimos para el desarrollo de plantaciones dendroenergéticas bajo condiciones edafoclimáticas específicas. Sin embargo, existen evidencias que híbridos del género Populus tendrían un gran potencial para estos usos.

La captura de carbono en los suelos es una promesa para la mitigación del cambio climático, aunque aún hoy existe controversia acerca de la capacidad de almacenamiento de carbono en los suelos dedicados a cultivos dendroenergéticos. Como las mayores capturas ocurrirían en los suelos más pobres, marginales para la agricultura, se abren expectativas para que Chile pueda usar parte de la amplitud de suelos que han sido sobreexigidos por la agricultura durante décadas.

El mejoramiento genético y la biotecnología serán las principales disciplinas que permitirán el aumento de la productividad de las plantaciones energéticas.

En Chile se prevén serios problemas energéticos debido a la dependencia de combustibles fósiles. Sin embargo, el país tiene excelentes características de suelo y clima para la conformación de plantaciones dendroenergéticas de alto rendimiento que permitan sustentar económica y ambientalmente su transformación en biocombustibles.

\section{REFERENCIAS}

Abrahamson LP, TA Volk, RF Kopp, EH White, JL Ballard. 2002. Willow Biomass Producer's Handbook. Syracuse, State University of New York, College of Environmental Science and Forestry. $31 \mathrm{p}$

Baker J, W Broadfoot. 1979. A practical field method of site evaluation for commercially important southern hardwoods.
General Technical Report SO-26. U.S. Department of Agriculture. Southern Forest Experiment Station. 51 p.

Balsari P, M Manzone, G Airoldi. 2007. Meccanizzazione dell'impianto e degli interventi coltutali nelle SRF. 4ta edizione FORLENER 07. Progetto Bioenerlegno: ottimizzazione della filiera di produzione di biomasse ligno-cellulosiche. Consultado 12 de may. 2008. Disponible en http://www. forlener.it/166_0/default.ashx.

BIOFRAC (Biofuels Research Advisory Council, EU). 2006. Biofuels in the European Union - A Vision for 2030 and Beyond. 32 p. (Final Draft Report of the Biofuels Research Advisory Council).

Brown K, R Driessche van den. 2002. Growth and nutrition of hybrid poplars over 3 years after fertilization at planting. Canadian Journal of Forest Research 32:226-232.

Campino J. 2006. Disponibilidad de biomasa en Chile. Concepción: Seminario Generación de Energía con Biomasa. FINPROLIGNUM. Consultado 15 de ago. 2008. Disponible en http://www.lignum.cl/doc/SeminarioBiomasaFINPRO_ LIGNUM.pdf.

Camps M, F Marcos. 2002. Los biocombustibles. Madrid, España. Mundi-Prensa. 383 p.

Carle J, Q Ma. 2005. Challenges of translating science into practice: poplars and other species in the Three North Region of China. Unasylva 56(221): 31-37.

Ceulemans R, RF Stettler, TM Hinckley, JG Isebrands JG, PE Heilman. 1990. Crown architecture of Populus clones as determined by branch orientation and branch characteristics. Tree Physiology 7: 157-167.

CNE (Comisión Nacional de Energía, CL). 2008. Balance Nacional de Energía 2007. Consultado 15 de ago. 2008. Disponible en http://www.cne.cl.

CNE (Comisión Nacional de Energía, CL), GTZ (Deutsche Gesellschaft für Technische Zusammenarbeit, DE), INFOR (Instituto Forestal, CL). 2007. Residuos de la industria primaria de la madera. Disponibilidad para uso energético. Santiago, Chile. 120 p.

Coleman M, JG Isebrands, DN Tolsted, V Tolbert. 2004a. Comparing Soil Carbon of Short Rotation Poplar Plantations with Agricultural Crops and Woodlots in North Central United States. Environmental Management 33(1):299-308.

Coleman MD, DR Coyle, J Blake, K Britton, M Buford, RG Campbell, J. Cox, B Cregg, D Daniels, M Jacobson et al. 2004b. Production of Short-Rotation Woody Crops Grown with a Range of Nutrient and Water Availability: Establishment Report and First-Year Responses. Asheville, NC 28802: U.S. Department of Agriculture, Forest Service. 21 p.

Coleman M, D Tolsted, T Nichols, WD Johnson, EG Wene, T Houghtaling. 2006. Post-establishment fertilization of Minnesota hybrid poplar plantations. Biomass and Bioenergy 30: 740-749.

Corcuera L, C Maestro, E Notivol. 2005. La ecofisiología como herramienta para la selección de clones más adaptados y productivos en el marco de una selvicultura clonal con chopos. Investigación Agraria: Sistemas y Recursos Forestales 14(3): 394-407.

Crow P, TJ Houston. 2004. The influence of soil and coppice cycle on the rooting habit of short rotation poplar and willow coppice. Biomass and Bioenergy 26: 497-505.

Dal Re L, S Bolognesi, A Innocenti. 2007 Pioppo da biomassa, i primi test sono positivi. Agricoltura: 67-69. Consultado 15 
dic. de 2008. Disponible en http://www.ermesagricoltura. it/Informazioni/Agricoltura.

DeBell DS, CA Harrington, GW Clendenen, MA Radwan, JC Zasada. 1997. Increasing the Productivity of Short-Rotation Populus Plantations. Olympia, Washington: USDA Forest Service. Pacific Northwest Research Station.

Deraedt W, R Ceulemans. 1998. Clonal variability in biomass production and conversion efficiency of poplar during the establishment year of a short rotation coppice plantation. Biomass and Bioenergy 15(4): 391-398.

Dimitriou I, P Aronsson. 2005. Sauces para energía y fitorremediación en Suecia. Unasylva 56(221): 47-50.

Dowell R, D Gibbins, J Rhoads, S Pallardy. 2008. Biomass production physiology and soil carbon dynamics in shortrotation-grown Populus deltoides and P. deltoides $x$ P.nigra hybrids. Forest Ecology and Management doi:10.1016/j. foreco.2008.08.023.

Emanuelli P. 2006. Biocombustibles sólidos en el sector forestal una aproximación a su potencialidad y estimación de beneficios. Documento Técnico No 178. CONAF. 12 p.

EU (European Union). 2006. Estrategia de la UE para los biocarburantes. Comunicación (2006) 34 Final. Comisión de las Comunidades Europeas. 31 p.

Facciotto G, A Giorgelli, L Vietto, G Allegro, G Castro, F Picco. 2006 Nuovi Cloni di Pioppo. Inserto. Agricoltura: 71-78. Consultado 15 dic. de 2008. Disponible en http://www. ermesagricoltura.it/Informazioni/Agricoltura

Facciotto G, G Mughini. 2007. Convegno progetto bioenerlegno. Ottimizzazione della filiera produzione di biomassa lignocellulosiche. FORLENER97. Consultado 12 de may. 2008. Disponible en http://www.forlener.it/166_0/default.ashx.

Farrell AE, RJ Plevin, BT Turner, AD Jones, M O'Hare, DM Kammen. 2006. Ethanol Can Contribute to Energy and Environmental Goals. Science 311: 506-508.

Gnansounou E, A Dauriat. 2005. Energy balance of bioethanol: a synthesis. 14th European Biomass Conference. Consultado 18 de ago. 2008. Disponible en http://www.eners.ch.

Gochis D, R Cuenca. 2000. Plant water use and crop curves for hybrid poplars. Journal of irrigation and drainage engineering 126(4): 206-214.

Hansen E. 1993. Soil carbon sequestration beneath hybrid poplar plantations in the North Central United States. Biomass and Bioenergy 5: 431-436.

Hansen E, R McLaughlin, P Pope. 1988. Biomass and nitrogen dynamics of hybrid poplar on two different soils: Implication for fertilization strategy. Canadian Journal of Forest Research 18(2): 223-230.

Hansen E, L Moore, D Netzer. 1983. Establishing intensively cultured hybrid poplar plantations for fuel and fiber. Gen. Tech. Rep. NC-78. St. Paul, MN: U.S. Department of Agriculture, Forest Service, North Central Forest Experimental Station. 24 p.

Hansen E, D Netzer. 1985. Weed control using herbicides in short-rotation intensively cultured poplar plantations. St. Paul, MN: US Department of Agriculture, Forest Service, North Central Forest Experiment Station. 6 p.

Ince P. 1977. Estimating effective heating value of wood or bark fuels at various moisture contents. Madison, Wisconsin: FPL 13. Forest Products Laboratory. U.S. Department of Agriculture. 9 p.

Johnson JM, MD Coleman, R Gesch, A Jaradat, R Mitchell, D Reicosky, WW Wilhelm. 2007. Biomass-Bioenergy Crops in the United States: A Changing Paradigm. The Americas Journal of Plant Science and Biotechnology 1(1): 1-28.

Kauter D, I Lewandowski, W Claupein. 2003. Quantity and quality of harvestable biomass from Populus short rotation coppice for solid fuel use - a review of the physiological basis and management influences. Biomass and Bioenergy 24: 411-427.

Madison M, G Brubaker. 1996. Water Resource Planning Considerations for Irrigated Short Rotation Intensive Culture Projects. 1st Conference of the Short Rotation Woody Crops Operations Working Group. Consultado 21 de ago. 2008. Disponible en http://www.woodycrops.org.

Marcos F. 2006. Generación de energía eléctrica con biomasa a medio y largo plazo. Anales de mecánica y electricidad: 22-27.

Martínez E, JP Fuentes, E Acevedo. 2008. Carbono orgánico y propiedades del suelo. Revista de la ciencia del suelo $y$ nutrición vegetal 8(1): 68-96.

May-de Mio L, L Amorin, E Moreira. 2006. Progresso de epidemias e avaliação de danos da ferrugem em clones de álamo. Fitopatologia Brasileira 31(2): 133-139.

Miller RO, P Bloese. 2002. Imazaquin and pendimethalin use for weed control in hybrid Poplar plantations in Michigan: secondyear results. Michigan State University East Lansing, MI 48824: Upper Peninsula Tree Improvement Center. 8 p.

Monclus R, E Dreyer, M Villar, FM Delmotte, D Delay, JM Petit, C Barbaroux, DL Thiec, C Bréchet, F Brignolas. 2006. Impact of drought on productivity and water use efficiency in 29 genotypes of Populus deltoides $\times$ Populus nigra. New Phytologist 169: 765-777.

Montoya JM. 1988. Chopos y choperas. Madrid: Mundi-Prensa. $122 \mathrm{p}$.

Nardin F. 2007. Use of selected fast growth poplar trees for biomass production in Europe. Opening Ceremony Poplar Technology Center University of Talca. Talca, Chile.

Netzer D, E Hansen. 1992. Seasonal variation in hybrid poplar tolerance to gliphosate. Res. Note NC-311 St. Paul, MN: US Department of Agriculture, Forest Service, North Central Forest Experiment Station. 7 p.

Orlovic S, V Guzina, B Krstic, L Merkulov. 1998. Genetic variability in anatomical, physiological and growth characteristics of hybrid poplar (Populus x euroamericana Dode (Guinier)) and Eastern cottonwood (Populus deltoids Bartr.) clones. Silvae genetica 47: 183-190.

Pari L. 2007. La logistica delle SRF: situazione attuale e prospettive future. 4ta edizione FORLENER 07. Progetto Bioenerlegno: ottimizzazione della filiera di produzione di biomasse lignocellulosiche. Consultado 12 de may. 2008. Disponible en http://www.forlener.it/166_0/default.ashx.

Perlack RD, LL Wright, AF Turhollow, RL Graham, BJ Stokes, DC Erbach. 2005. Biomass as Feedstock for a Bioenergy and Bioproducts Industry: The Technical Feasibility of a BillionTon Annual Supply. Oak Ridge National Laboratory. 78 p.

Pinon J, P Frey, C Husson. 2006. Wettability of Poplar Leaves Influences Dew Formation and Infection by Melampsora larici-populina. Plant disease 90(2): 177-184.

Pontailler JY, R Ceulemans, J Guittet. 1999. Biomass yield of poplar after five 2-year coppice rotations. Forestry 72(2): 157-163.

Rae AM, KM Robinson, NR Street, G Taylor. 2004. Morphological and physiological traits influencing biomass productivity in 
short-rotation coppice poplar. Canadian Journal of Forest Research 34: 1488-1498.

Ragauskas AJ, CK Williams, BH Davison, G Britovsek, J Cairney, CA Eckert, JP Hallett, DJ Leak, CL Liotta, JR Mielenz, R Murphy, R Templer, T Tschaplinski. 2006. The Path Forward for Biofuels and Biomaterials. Science 311: 484-489.

Ramírez CC, F Zamudio, J Verdugo, ME Núñez. 2004. Differential susceptibility to the aphid Chaitophorus leucomelas of poplar hybrids recently introduced to Chile. Journal of Economic Entomology 97: 1965-1971.

Riemenschneider DE, WE Berguson, DI Dickman, RB Hall, JG Isebrands, CA Mohn, R Stanosz, GA Tuskan. 2001. Poplar breeding and testing strategies in the north-central U.S.: Demonstration of potential yield and consideration of future research needs. The Forestry Chronicle 77(2): 245-253.

Robert M. 2002. Captura de carbono en los suelos para un mejor manejo de la tierra: Informes sobre recursos mundiales de suelos $\mathrm{N}^{\mathrm{o}}$ 96. FAO. 69 p.

Rutz D, R Janssen. 2008. Biofuel Technology Handbook. Sylvensteinstr 2. München Germany: WIP Renewable Energies. $152 \mathrm{p}$.

Schlatter J, V Gerding. 2001. Balance de carbono en suelos arenosos con plantaciones de Pinus radiata en Chile central. Simposio Internacional Medición y Monitoreo de la Captura de Carbono en Ecosistemas Forestales. Consultado 21 de ago. 2008. Disponible en http://www.uach.cl/procarbono.

Shock CC, EB Feibert, LD Saunders, M Seddigh. 2002. Initial Growth of Irrigated Hybrid Poplar Decreased by Ground Covers Western. Journal of Applied Forestry 17(2): 61-65.

Sixto H, M Hernández, M Barrio, J Carrasco, I Cañellas. 2007. Plantaciones del género Populus para la producción de biomasa con fines energéticos: revisión. Investigación Agraria: Sistemas y Recursos Forestales 16(3): 277-294.

Smart L, T Volk, J Lin, R Kopp, I Phillips, K Cameron, E Whit, L Abrahamson. 2005. Mejora genética de los cultivos de sauce (Salix spp). con fines bioenergéticos y medioambientales en los Estados Unidos. Unasylva 56(221): 51-55.

Souch CA, W Stephens. 1998. Growth, productivity and water use in three hybrid poplar clones. Tree Physiology 18: 829-835.

Sperandio G. 2007. Aspetti economici delle colture da biomassa a ciclo breve - Seconda parte: redditività dell'investimento. 4ta edizione FORLENER 07. Progetto Bioenerlegno: ottimizzazione della filiera di produzione di biomasse ligno-cellulosiche. Consultado 12 de may. 2008. Disponible en http://www.forlener.it/166_0/default.ashx.

Spiers A, D Hopcroft. 1988. Penetration and infection of poplar leaves by urediniospores of Melampsora larici-populina and Melampsora medusae. New Zealand Journal of Botany 26: 101-111.

Stanton B, J Eaton, J Johnson, D Rice. 2002. Hybrid poplar in the Pacific Northwest: The effects of market-driven management. Journal of Forestry 100(4): 28-33.

Stanton BJ. 2001. Clonal variation in basal area growth patterns during stand development in hybrid poplar. Canadian Journal of Forest Research 31: 2059-2066.
Stanturf JA, C Oosten van, DA Netzer, MD Coleman, CJ Portwood. 2001. Ecology and silviculture of poplar plantations. Poplar Culture in North America. Part A, Chapter 5. Edited by D.I. Dickmann, J.G. Isebrands, J.E. Eckenwalder, and J. Richardson ed. Ottawa, ON KlA OR6, Canada. p 153-206.

Swift R. 2001. Sequestration of carbon by soil. Soil Science 166(11): 858-871.

Thomas K, P Comeau, K Brown. 2000. The silviculture of hybrid poplar plantation. British Columbia, Ministry of Forest Research Program. 7 p.

Tuskan G, M Walsh. 2001. Short-rotation woody crop systems, atmospheric carbon dioxide and carbon management: A U.S. case study. Forestry Chronicle 77(2): 259-264.

Tuskan GA, S DiFazio, S Jansson, J Bohlmann, I Grigoriev, U Hellsten, N Putnam, S Ralph, S Rombauts, A Salamov, J Schein, L Sterck, A Aerts, RR Bhalerao, RP Bhalerao, D Blaudez, W Boerjan, A Brun, A Brunner, V Busov, M Campbell, J Carlson, M Chalot, J Chapman, D Cooper, PM Coutinho, J Couturier, S Covert, Q Cronk, R Cunningham, J Davis, S Degroeve, A Dejardin, C DePamphillis, J Detter, B Dirks, I Dubchak, S Duplessis, J Ehiting, B Ellis, K Gendler, D Goodstein, M Gribskov, J Grimwood, A Groover, L Gunter, B Hamberger, B Heinze, Y Helariutta, B Henrissat, D Holligan, R Holt, W Huang, N Islam-Faridi, S Jones, M Jones-Rhoades, R Jorgensen, C Joshi, J Kangasjarvi, J Karlsson, C Kelleher, R Kirkpatrick, M Kirst, A Kohler, U Kalluri, F Larimer, J Leebens-Mack, P Locascio, Y Lou, S Lucas, F Martin, B Montanini, C Napoli, DR Nelson, D Nelson, K Nieminen, O Nilsson, G Peter, R Philippe, G Pilate, A Poliakov, J Razumovskaya, P Richardson, C Rinaldi, K Ritland, P Rouze, D Ryaboy, J Schmutz, J Schrader, B Segerman, H Shin, A Siddiqui, F Sterky, A Terry, C Tsai, E Uberbacher, P Unneberg, J Vahala, K Wall, S Wessler, G Yang, T Yin, C Douglas, M Marra, G Sandberg, D Rokhsar. 2006. The Genome of Black Cottonwood, Populus trichocarpa (Torr. et Gray). Science 313: 1596-1604.

Volk TA, T Verwijst, PJ Tharakan, LP Abrahamson, EH White. 2004. Growing fuel: a sustainability assessment of willow biomass crops. Frontiers in Ecology and the Environment 2(8): 411-419.

Welham C, K Rees van, B Seely, H Kimmins. 2007. Projected long-term productivity in Saskatchewan hybrid poplar plantations: weed competition and fertilizer effects. Canadian Journal of Forest Research 37(2): 356-370.

Widin KD, AL Schipper. 1981. Epidemiology and impact of Melampsora medusae leaf rust infection on yield of hybrid poplars in north-central United States. European Journal of Plant Pathology 11: 438-488.

Yu Q. 2001. Can physiological and anatomical characters be used for selecting high yielding hybrid aspen clones? Silva Fennica 35: 137-146.

Zamudio F, R Baettig, F Guerra. 2008. Origen y futuro del cultivo del álamo en Chile. Monografía Proyecto FONDEF D04I1027. Talca, Chile. Universidad de Talca. 34 p.

Recibido: 17.12.08 Aceptado: 12.04.10 